\title{
Intraoperative Simulation and Planning Using a Combined Acetabular and Femoral (CAF) Navigation System for Total Hip Replacement
}

\author{
Yoshinobu Sato $^{1,4}$, Toshihiko Sasama ${ }^{4,1}$, Nobuhiko Sugano ${ }^{2}$, Kei Nakahodo ${ }^{4,1}$, \\ Takashi Nishii $^{2}$, Kenji Ozono ${ }^{3}$, Kazuo Yonenobu ${ }^{2}$, Takahiro Ochi ${ }^{3}$, and \\ Shinichi Tamura ${ }^{1,4}$ \\ 1 Division of Functional Diagnostic Imaging \\ 2 Department of Orthopaedic Surgery \\ ${ }^{3}$ Division of Computer Integrated Orthopaedic Surgery \\ Osaka University Graduate School of Medicine \\ 4 Department of Informatics and Mathematical Science \\ Graduate School of Engineering Science, Osaka University \\ yoshi@image.med.osaka-u.ac.jp, \\ http://www.image.med.osaka-u.ac.jp/ yoshi
}

\begin{abstract}
This paper describes intraoperative simulations for the limb length and the range of motion (ROM) adjustment in total hip relacement (THR) surgery, and their utility in intraoperative planning. After implantation of the cup and stem, final adjustments can be made to the limb length and ROM by selecting the optimal combination of femoral neck and head components from the range available in a changeable modular system. The aim of this work is to provide intraoperative assistance to the surgeon in selecting the optimal component combination as well as in planning additional osteotomy to remove unwanted bone impingements and widen the safe ROM. Using the positions and orientations of the cup and stem intraoperatively measured by a combined acetabular and femur (CAF) navigation system, limb length and ROM simulations are carried out for neck and head components of various lengths and angles. These simulations provide information on limb length, the ROM, and where in a 3D model impingements will occur for each combination of components. The accuracy of the simulations was evaluated by comparison with postoperative CT data for the limb length and actually measured motions for the ROM.
\end{abstract}

\section{Introduction}

In total hip replacement (THR), computer assisted navigation for placement of the acetabular cup has been shown to be highly useful [1, [2, 3]. Precise alignment of the cup orientation is regarded as particularly important to reduce the possibility of complications such as dislocation, wear, and loosening. Previous navigation systems for THR have consisted of the distinctly separate stages a preoperative planning and simulation stage and the intraoperative navigation 
itself [1], [2], [3]. In the preoperative stage, optimal parameters such as cup size, position, and orientation are determined through surgical planning that includes the range of motion (ROM) simulation [4] and the limb length adjustment. In the intraoperative stage, the actual position and orientation of the cup are accurately measured using an optical 3D sensor so as to execute exactly what was planned preoperatively. However, this two-stage approach is insufficient because the actual execution during the intraoperative stage often differs from the preoperative plan for the following reasons:

- Manual placement of the cup component is inherently inaccurate even if a sophisticated navigation system is utilized.

- The preoperative plan sometimes needs to be changed during the operation if conditions are encountered that were not anticipated from the preoperative images. For example, the cup position may need to be changed if the bone tissue around the preoperatively planned position is found to be unexpectedly fragile.

To address the above problems, our system incorporates additional intraoperative simulation and planning stages that are implemented after the two stages described above.

In this paper, we describe intraoperative simulations for limb length and ROM adjustment in THR. In our hospital, after implanting the acetabular cup and femoral stem, the surgeon makes final adjustments to the limb length and ROM by selecting the optimal combination of neck and head components from the range available in a changeable modular femoral head and neck system (ANCA-FIT, Cremascoli, Milan) (Fig. 1). The neck and head components vary in their length and angle (the depth of the socket in the case of the head component), and component selection has a significant effect on both the limb length and ROM. The surgeon performs additional osteotomy (bone cutting) to remove some bone regions so as to prevent the impingement of bone on bone or of the implant on bone, which would reduce the safe ROM (hereafter, the impingement of $\mathrm{A}$ on $\mathrm{B}$ is referred to as the $\mathrm{A}-\mathrm{B}$ impingement). The purpose of the intraoperative simulation is to assist the surgeon in intraoperative planning with regard to selecting the optimal combination of neck and head components and determining which additional bone regions should be removed by osteotomy. In our intraoperative simulation, the limb length and ROM are simulated for neck and head components with various lengths and angles based on the positions and orientations of the acetabular cup and the femoral stem implants, which are intraoperatively measured after implantation using a combined acetabular and femur (CAF) navigation system for THR [5]. Intraoperative planning is then carried out based on the simulation results as to which neck and head components should be selected and where additional osteotomy should be performed to avoid unwanted impingements. 


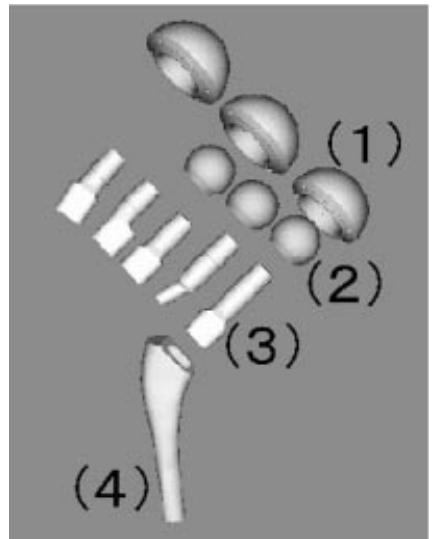

(a) Components

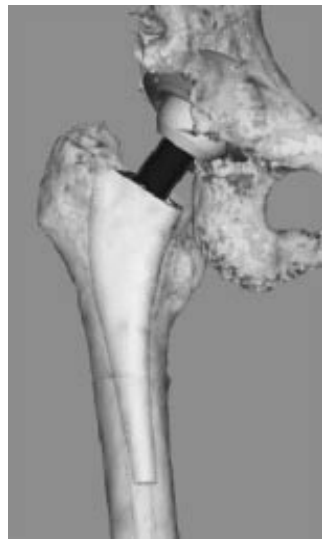

(b) Implantation

Fig. 1. Changeable modular femoral head and neck system. (1) Cup, (2) head, (3) neck, and (4) stem.

\section{Intraoperative Simulation and Planning for Total Hip Replacement}

\subsection{Combined Acetabular and Femur (CAF) Navigation System}

The combined acetabular and femur $(\mathrm{CAF})$ navigation system employed is an Optotrak-based guidance system for THR (Optotrak, Northern Digital Inc., Waterloo, Ontario, Canada) 5 . The novel feature of this CAF system is that it provides the guidance of both pelvis and femoral sides - unlike other systems which deal with either the acetabular [1, [2, 3] or femur [6] side. This feature enables the surgeon to intraoperatively evaluate the geometric and kinematic parameters inherent in the hip joint, including limb length and ROM as addressed in this paper. Using 3D surface models of the pelvis and femur reconstructed from preoperative CT images, surface-based registration is performed by applying the iterative closest point (ICP) algorithm for both the acetabular and femoral sides. To attain a high level of accuracy on each side, we evaluated the optimal sampling areas of 3D points on clinically available bony surfaces so as to balance accuracy and invasiveness [7. The placement of the cup and stem components is guided and measured using localizers to which Optotrak LED markers are attached (Fig. 2(a)). During the operation, the motions of both the pelvis and femur are tracked by means of rigid bodies attached to them (Fig. 2(b)). Another advantage of our CAF system in THR is that it provides guidance in osteotomy for femoral head resection on the femoral side.

\subsection{Pelvis- and Femur-Centered Coordinate Systems}

Using anatomical landmarks, the pelvis-centered and femur-centered coordinate systems (pelvis-CS and femur-CS) are preoperatively determined to measure the 


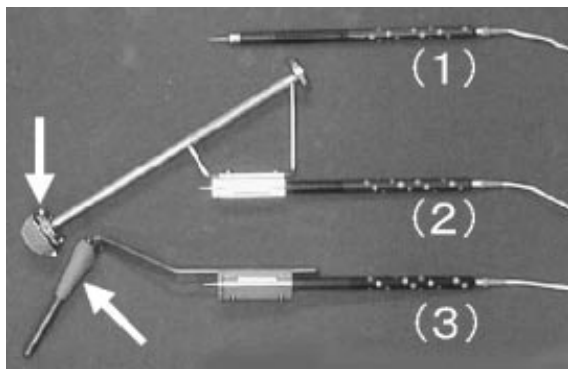

(a) Localizers

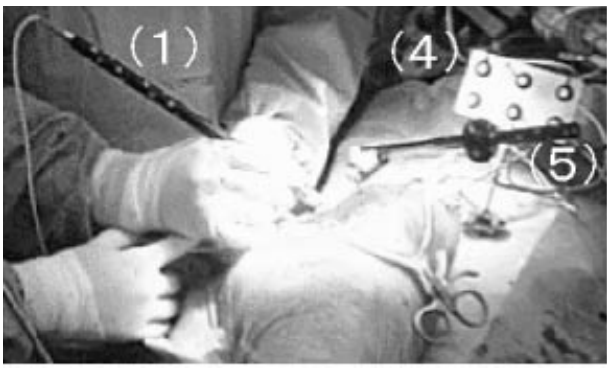

(b) Intraoperative use

Fig. 2. Localizers and trackers with LED markers for the CAF system and their intraoperative use. (1) Optotrak pen-probe, (2) cup localizer, (3) stem localizer, (4) pelvis tracker (rigid body attached to pelvis), and (5) femur tracker (rigid body attached to femur).

geometric and kinematic parameters inherent in the hip joint of each patient. Figure 3 shows the anatomy of the hip joint. The anatomical landmarks are localized on the 3D surface model of the pelvis and femur reconstructed from the CT images. The pelvis-CS and femur-CS are based only on the 3D shape inherent in the pelvis and femur, respectively.

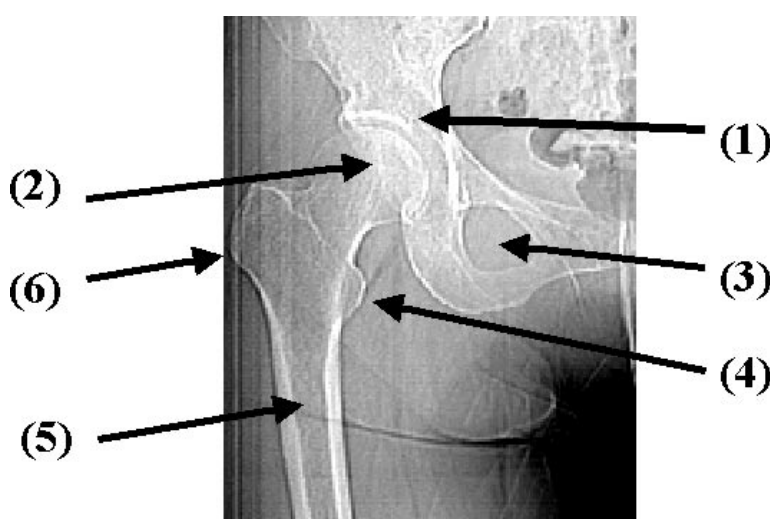

Fig. 3. Anatomy of the hip joint and anatomical landmarks. (1) Acetabulum, (2) femoral head, (3) foramen obturatum, (4) trochanter minor, (5) medullary space, and (6) trochanter major.

We define the pelvis-CS in the following manner. Firstly, the $x z$-plane of the pelvis-CS is defined. Consider a plane whose normal is vertical (like the top 
of a desk). A pelvis placed on the plane with its frontal side downward is in a stable pose supported by three points on the pelvis surface. The $x z$-plane of the pelvis-CS is then defined as the plane tangential to the frontal side of the pelvis as described above (Fig. 4(a)). Secondly, the $x y$-plane is defined as the plane tangential to the upper rims of the two holes in the pelvis (the foramina obturata, see Fig. 3) and orthogonal to the $x z$-plane. Finally, the $y z$-plane is defined as the plane passing through the center of the bridge between the left and right parts of the pelvis (the symphysis pubica) and orthogonal to both the $x z$-plane and the $x y$-plane (Fig. 4(b)).

We define the femur-CS in following manner. Firstly, the $x z$-plane of the femur-CS is defined as the plane tangential to the posterior side of the femur in a manner analogous to that described above for the pelvis-CS (Fig. 4(c)). Secondly, the $z$-axis is defined as the orthogonal projection of the medullary axis, which is the principal axis of the medullary space (see Fig. 3), on the $x z$ plane. Thirdly, the origin is defined as the orthogonal projection of the crown position of the trochanter minor, a prominent anatomical landmark (see Fig. 3) on the $x z$-plane. Finally, the $x y$-plane is defined as the plane passing through

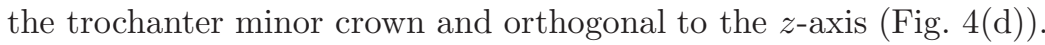

\subsection{Representation of Positions and Orientations of Cup and Stem}

The CT image coordinate system (image-CS) and the Optotrak coordinate system (Optotrak-CS) are both unsuitable for representing the geometric and kinematic properties inherent in the hip joint. In order to measure and analyze these properties, we need to determine the positions and orientations of the cup and stem in the pelvis-CS and femur-CS, respectively.

For this purpose, we employ cup-centered and stem-centered coordinate systems (cup-CS and stem-CS) in which the 3D shapes of the cup and stem are respectively represented. We define the cup-CS in the following manner. First, the origin of the cup-CS is defined as the center of the spherical surface of the cup hemisphere. The $z$-axis of the cup-CS is then defined as the normal of the plane fitted to the cup rim. Since the cup implant we use is rotationally symmetric around the $z$-axis, the $x$-axis and $y$-axis are not determined. The stem-CS is similarly uniquely defined based on the rim shape and orientation of the socket into which the modular neck component is inserted (the details are not described here).

Let $T_{i o}$ be a $4 \times 4$ matrix representing the transformation from the image-CS to the Optotrak-CS, which is estimated during the intraoperative registration stage; let $T_{o c}$ be the transformation from the Optotrak-CS to the cup-CS, which is estimated by combining the intraoperatively measured 3D positions of the cup localizer with the preoperative tool calibration; and let $T_{p i}$ be the transformation from the pelvis-CS to the image-CS, which is estimated during the preoperative planning stage. What we need is $T_{p c}$, representing the cup position and orientation in the pelvis-CS. We obtain the transformation from the pelvis-CS to the cup-CS, $T_{p c}$, using the above transformations as follows:

$$
T_{p c}=T_{p i} T_{i o} T_{o c}
$$




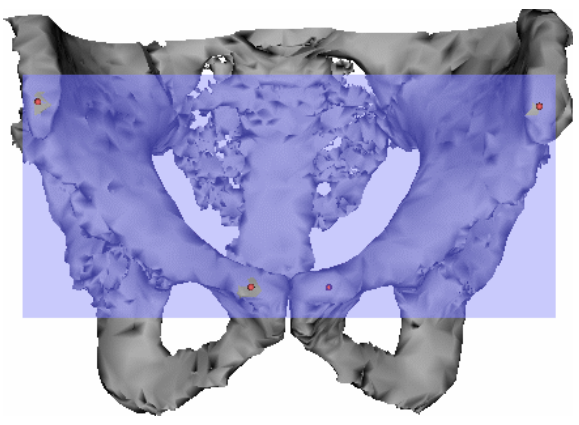

(a)

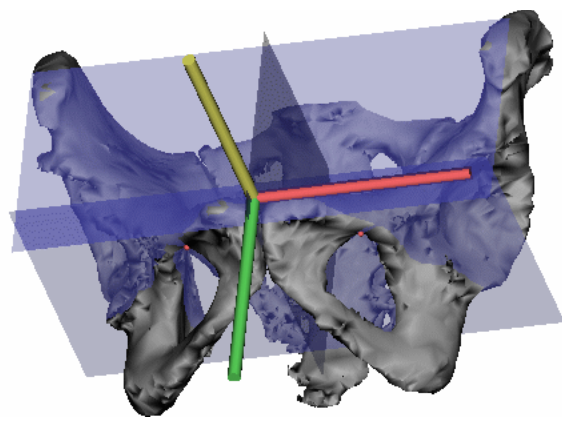

(b)

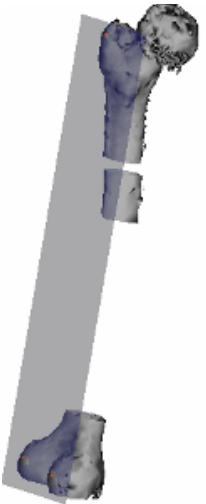

(c)

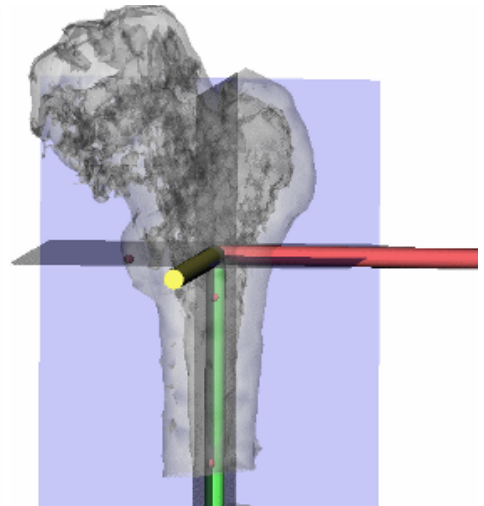

(d)

Fig. 4. Pelvis-centered and femur-centered coordinate systems (Pelvis-CS and femur CS). (a) The $x z$-plane with the pelvis 3D model. (b) Pelvis-centered coordinate system. (c) The $x z$-plane with the femur 3D model. (d) Femur-centered coordinate system.

Similarly, we obtain the transformation from the femur-CS to the stem-CS, $T_{f s}$, as follows:

$$
T_{f s}=T_{f i} T_{i o} T_{o s}
$$

where $T_{o s}$ and $T_{f i}$ are the transformations from the Optotrak-CS to the stem-CS and the femur-CS to the image-CS, respectively.

\subsection{Representation of Hip Joint Motion}

After implanting the cup and stem, the surgeon selects the most appropriate combination of neck and head components from those available in the changeable modular femoral head and neck system (Fig. 1) to adjust the limb length and ROM. 
This combination determines the transformation from the cup-CS to the stem-CS, $M_{c s} . M_{c s}$ is also a $4 \times 4$ matrix representing the transformation, which can decomposed as

$$
M_{c s}=T_{c s} R_{c s}
$$

where $T_{c s}$ is a fixed transformation determined by the combination of the neck and head components. $R_{c s}$, which represents the hip joint motion, is a variable transformation but constrained to the rotational motion whose center is the origin of the cup-CS. The overall hip joint motion is represented by the transformation from the pelvis-CS to the femur-CS, $M_{p f}$, which is given by

$$
M_{p f}=T_{p c} T_{c s} R_{c s} T_{s f}=T_{p c} T_{c s} R_{c s} T_{f s}^{-1},
$$

where $R_{c s}$ is variable due to the hip joint motion and $T_{c s}$ is changeable according to the combination of head and neck components selected.

\subsection{Limb Length Simulation}

Minimizing limb length discrepancy between left and right is of great importance in THR. In order to measure the limb length, we first define the normalized arrangement of the hip joint. In the normalized arrangement, $R_{c s}$, which is a variable part of the transformation representing the rotational motion of hip joint, is determined so that the directions of the three coordinate axes of the pelvis-CS are the same as those of the femur-CS. We then define the limb length as the projective length of $\overrightarrow{O_{p} O_{f}}$ on the $z$-axis in the normalized arrangement, where $O_{p}$ and $O_{f}$ are the origins of the pelvis-CS and femur-CS, respectively. This length corresponds to the distance along the $z$-axis between the upper rim of the foramen obturatum on the pelvis and the crown of the trochanter minor on the femur in the normalized arrangement, which are anatomical landmarks used in determining the pelvis-CS and femur-CS. The limb length simulation for testing different combinations of neck and head components is realized by changing the transformation $T_{c s}$ (in Equation (41)), which corresponds to each combination. Since $T_{p c}$ (from the pelvis to the cup) and $T_{s f}$ (from the stem to the femur) needed to measure the limb length are obtained intraoperatively, this simulation is performed intraoperatively.

With respect to the other side of the leg (which is assumed not to be an implant), the limb length is preoperatively measured using the method described above. The femur-CS is determined by the same method. The pelvis-CS is common to both the left and right legs. $T_{p c}$ (from the pelvis to the cup) and $M_{c f}$ (from the cup to the femur) are measured from CT images, where $M_{c f}=T_{c f} R_{c f}$. The origin of the cup-CS is determined as the center of the sphere approximating the femoral head in CT images. The orientation of the cup-CS does not affect the limb length since it is measured in the normalized arrangement. $R_{c f}$ is determined so that the the directions of the three axes of the pelvis-CS are the same as those of the femur-CS. Figure 5 shows examples of the intraoperative simulation for limb length adjustment. 

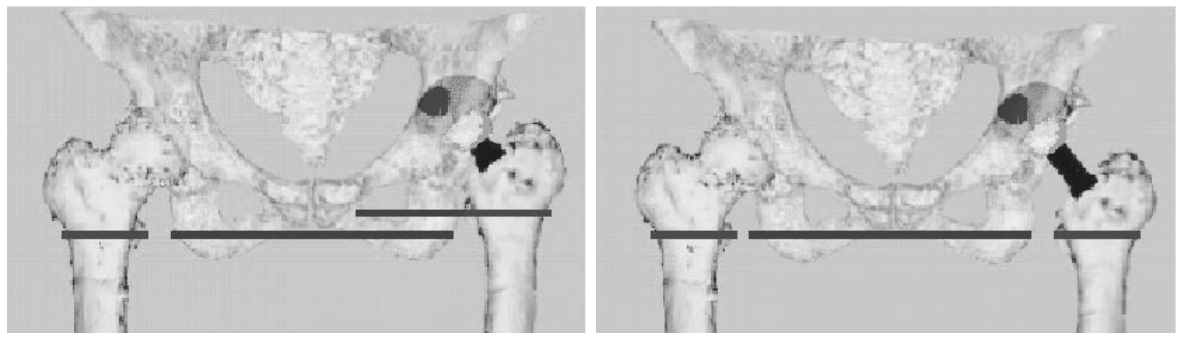

Fig. 5. Intraoperative simulation for limb length adjustment. Left: A large discrepancy between left and right is observed. Right: The discrepancy is minimized by changing the neck component.

\subsection{Range of Motion (ROM) Simulation}

The range of motion (ROM) is regarded as the intersection of sets of ROMs estimated on the basis of implant-implant, bone-implant, and bone-bone impingements (Fig. (6)). In the hip joint motion shown in Equation (4), $T_{c s}$ can be changed using different combinations of neck and head components. As described earlier, $R_{c s}$ is the rotational motion whose center is the origin of the cup-CS. The range of rotational angles in $R_{c s}$ is constrained by the impingements mentioned above, and the safe ROM can be regarded as the range of rotation angles without such impingements. $R_{c s}$ can be decomposed into the direction $\overrightarrow{O_{c} O_{s}}$ and the rotation around $\overrightarrow{O_{c} O_{s}}$. Here, we assume that the rotation around $\overrightarrow{O_{c} O_{s}}$ is fixed and we estimate the range of the direction $\overrightarrow{O_{c} O_{s}}$ without impingements. A collision detection algorithm (V-CoLLIDE, http://www.cs.unc.edu/ geom/V_COLLIDE) is employed to find the ROM using the CT surface models of the pelvis and femur, and the CAD surface models of the implants. In the ROM simulation, the intraoperatively measured positions and orientations of the cup and stem $\left(T_{p c}\right.$ and $\left.T_{s f}\right)$ are used. The ROM simulation for testing the different combinations of neck and head components is realized by changing the transformation $T_{c s}$ Thus, the ROM simulation results suggest the best combination of neck and head components.

Using the collision detection algorithm, the surgeon can also determine where in the 3D models collisions (impingements) occur and the extent to which they reduce the ROM. Hence, the ROM simulation results also suggest where bone should be removed by osteotomy to widen the safe ROM.

\section{Results}

We applied the procedures described in Section 2 to ten cases of THR at Osaka University Hospital. Postoperative CT images were obtained to compare intraoperatively measured parameters with those estimated from the postoperative images. Both the preoperative and postoperative CT images $(512 \times 512$ matrix $)$ 

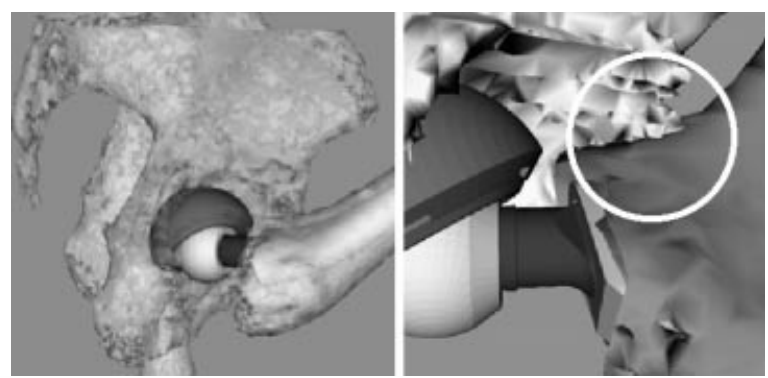

Fig. 6. Impingement of bone on bone in intraoperative ROM simulation.

were obtained with a 3 -mm slice thickness, $3-\mathrm{mm}$ reconstruction pitch, and 420 mm FOV.

\subsection{Limb Length Simulation}

We evaluated the intraoperatively estimated limb length by comparing it with that estimated using postoperative CT images. The postoperative images were processed, and the postoperative cup position, cup orientation, and limb length were measured, as follows. Firstly, the CT surface models of the pelvis and femur were reconstructed from the postoperative CT images. The postoperative pelvis model was registered to the preoperative pelvis model using the ICP algorithm and the same pelvis-CS as the preoperative one was determined. Secondly, the position and orientation of the implanted cup (the cup-CS) in the pelvis-CS were estimated by fitting the CAD model of the cup. In this way, the center of the rotation $R_{c f}$ was found. Finally, the femur-CS in the postoperative model was defined and its orientation was aligned to the pelvis-CS so that the pelvis and femur models were positioned in the normalized arrangement. The postoperative limb length was then measured.

Table 1 shows the differences between the intraoperative and postoperative results for the cup position, cup orientation, and limb length, which taken together can be regarded as a good approximation of the overall accuracy of the method. For the cup position and orientation, the distance and difference in angle, which are absolute values, were respectively used as the error measures. For the limb length, the difference between the two results, which is a signed value, was used. The root-mean-squares (RMS) were around $3 \mathrm{~mm}$ and $4 \mathrm{de}-$ grees. Intraoperative adjustment of limb length in gradations of every 2 or 3 $\mathrm{mm}$ is possible by changing the neck and head component combinations. The accuracy of the limb length simulation was regarded as acceptable considering that the differences included the registration error between the preoperative and postoperative pelvis models. 


\subsection{ROM Simulation}

Figure 7 shows the ROM results for two cases (\#6 and \#7). These were plotted based on the implant-implant impingement (labeled "implant" in Fig. 7) and all possible impingements (labeled "simulation"). The ROM is represented by the $z$-axis direction of the femur-CS in the pelvis-CS. The radial directions $0^{\circ}$ and $90^{\circ}$ correspond to the left and frontal directions, respectively. We also plotted the directions of the femur relative to the pelvis measured intraoperatively by actual ROM testing - i.e. not by simulation - using the Optotrak system (labeled "real"). These measurements were made while the surgeon moved the leg in the frontal directions. In each case, the same combination of neck and head components was used for the actual and simulated measurements. The ranges of movement simulated intraoperatively were consistent with those actually measured.

In the case shown in Fig. 77(a), a ROM simulation was also performed with a different combination of neck and head components from that used for the actual measurement. The result, shown in the left frame, confirmed that the ROM could be markedly altered by changing the component combination.

In the case shown in Fig. 7(b), the surgeon performed additional osteotomy on the pelvic bone to remove unwanted impingements and widen the safe ROM. We intraoperatively reconstructed the bone shape resurfaced by the additional osteotomy based on a set of 3D points obtained by digitizing the bone surface using the Optotrak pen-probe [8]. ROM simulations were done using the pelvis models obtained before and after the additional osteotomy (respectively labeled "simulation 1" and "simulation 2"). The results confirmed that the ROM was widen by the additional osteotomy.

\section{Discussion and Conclusions}

We have described intraoperative limb length and range of motion (ROM) simulations in total hip replacement (THR) surgery and demonstrated their usefulness in intraoperative planning to select the best combination of neck and head components in a changeable modular system as well as in additional osteotomy to widen the safe ROM. In order to measure the geometric and kinematic properties inherent in the hip joint, the pelvis- and femur-centered coordinate systems were preoperatively determined. The positions and orientations of the acetabular

Table 1. Accuracy evaluation of intraoperatively measured cup position, orientation, and limb length.

\begin{tabular}{|c||c|c|c|c|c|c|c|c|c|c||c|}
\hline Case \# & 1 & 2 & 3 & 4 & 5 & 6 & 7 & 8 & 9 & 10 & RMS \\
\hline Cup position $(\mathrm{mm})$ & 2.96 & 1.50 & 4.38 & 3.05 & 3.40 & 3.55 & 1.15 & 2.08 & 3.79 & 4.64 & 2.98 \\
\hline Cup orientation (degree) & 4.55 & 2.84 & 3.38 & 1.84 & 6.60 & 4.91 & 1.91 & 2.67 & 3.28 & 1.52 & 4.25 \\
\hline Limb length (mm) & 6.35 & 0.50 & -0.84 & 3.53 & 1.11 & -4.45 & -0.18 & 3.12 & -2.21 & -5.11 & 3.40 \\
\hline
\end{tabular}


cup and femoral stem in these coordinate systems were then obtained intraoperatively using a combined acetabular and femur (CAF) navigation system for utilization in the simulations based on the intraoperative conditions of the hip joint.

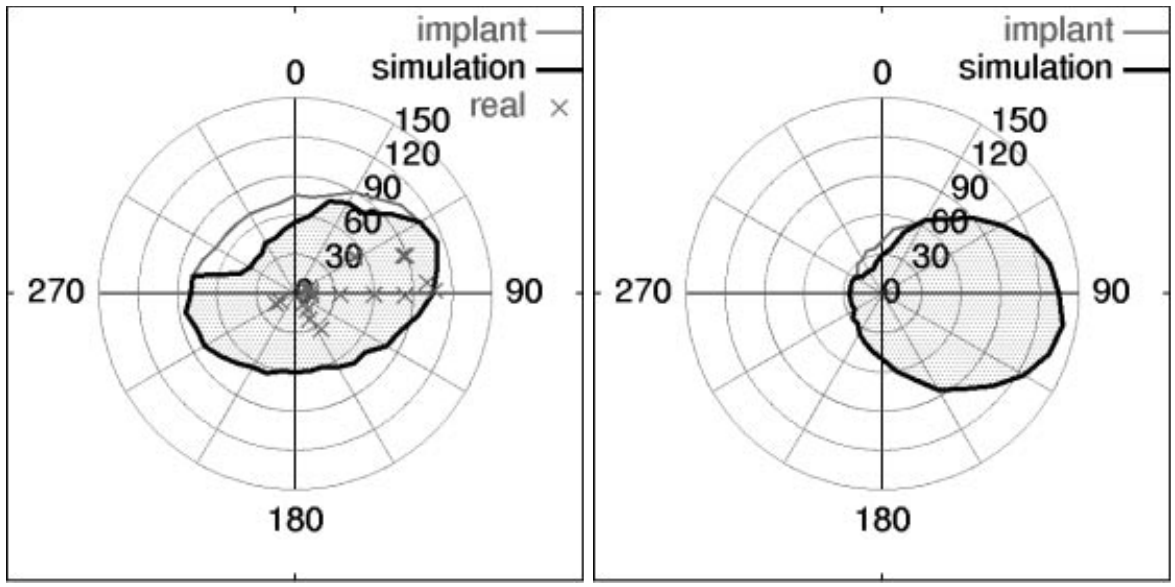

(a) Case 6

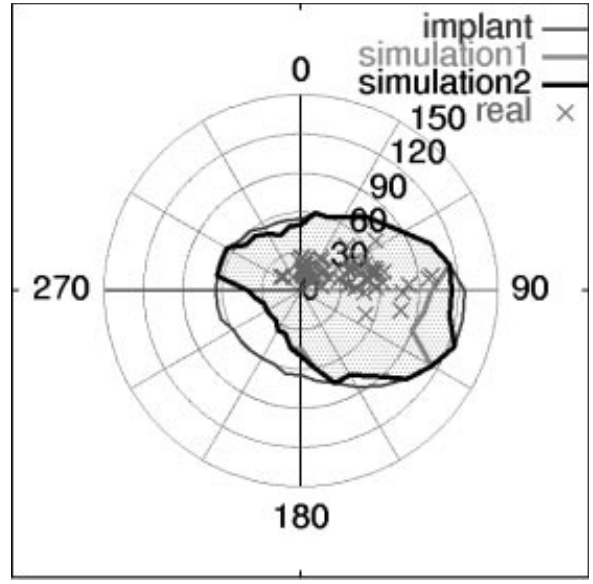

(b) Case 7

Fig. 7. Intraoperative ROM simulation results. See text for details.

These intraoperative simulations offer two distinct advantages. Firstly, the most suitable combination of head and neck components can be selected based on comprehensive simulation results. This is advantageous for the surgeon because it is difficult to actually measure the motion limits in various directions or the limb length for various combinations of head and neck components during the operation. Secondly, using 3D models the surgeon can easily determine impingement locations and where additional osteotomy should be performed. 
In clinical trials, the accuracy of intraoperatively estimated limb lengths was evaluated by comparison with postoperative CT images, and was found to be acceptable. The ROM obtained by intraoperative simulation was compared with actually measured motions and confirmed to be consistent with them. While the experimentally evaluated overall accuracy was acceptable, it was apparent that the overall accuracy was liable to be affected by several potential sources of error including tool calibration and registration [7] as well as the determination of the pelvis- and femur-centered coordinate systems. Furthermore, error sources on the pelvis side are combined with those on the femur side in the hip joint simulation. Hence, in future work we will consider how the theory of error propagation [9, 10 can be applied to determine the extent to which each error source might affect the overall accuracy.

Since our work is focused on the uniqueness of the geometric and kinematic parameters of the hip joint, in defining the pelvis- and femur-centered coordinate systems it was our intention that these coordinate systems should be uniquely determined based on their 3D shapes. Although the coordinate systems used in this study provided unique parameters, they should be improved so as to describe geometric and kinematic properties that can be directly related to the joint function. For example, in the present system the $z$-axis of the pelvis-centered coordinate system does not correspond to any functional axis of hip joint motion. In future work, such factors need to be incorporated when defining the coordinate systems [11].

Acknowledgment This work was partly supported by the Japan Society for the Promotion of Science (JSPS Research for the Future Program).

\section{References}

[1] Digioia AM III, Jaramaz B, Blackwell M, et al.: Image guided navigation system to measure intraoperatively acetabular implant alignment, Clinical Orthopaedics and Related Research, 355, 8-22 (1998).

[2] Jaramaz B, Digioia AM III, Blackwell M, et al.: Computer assisted measurement of cup placement in total hip replacement, Clinical Orthopaedics and Related Research, 354, 70-81 (1998).

[3] Langlotz U, Lawrence J, Hu Q, et al.: Image guided cup placement, Computer Assisted Radiology and Surgery (CAR'99), Paris, 717-721 (1999).

[4] Jaramaz B, Nikou C, Digioia AM III, et al.: Effect of cup orientation and neck length in range of motion simulation, Proc. 43rd Annual Meeting of the Orthopaedics Research Society, 186 (1997).

[5] Sugano N, Sato Y, Sasama T, et al.: Combined acetabular and femoral surgical navigation in total hip arthroplasty, Computer Assisted Radiology and Surgery (CAR'99), Paris, 722-725 (1999).

[6] Taylar RH, Mittelstadt BD, Paul HA, et al.: An image-directed robotic system for precise orthopaedic surgery, IEEE Transactions on Robotics and Automation, 10(3), 261-275 (1994).

[7] Sasama T, Sato Y, Sugano N, et al.: Accuracy evaluation in computer assisted hip surgery, Computer Assisted Radiology and Surgery (CAR'99), Paris, 772-776 (1999).

[8] Nakahodo K, Sasama T, Sato Y, et al.: Intraoperative update of 3-D bone model during computer navigation of pelvic osteotomies using real-time 3-D position data, Computer Assisted Radiology and Surgery (CARS2000), San Francisco (2000).

[9] Lea J and Peshkin M: Registration graphs: a diagramming and analysis tool for registration in computer-assisted surgery, Computer Assisted Radiology and Surgery (CAR'99), Paris, $767-771$ (1999).

[10] Smith RC and Cheeseman P: On the representation and estimation of spatial uncertainty, International Journal of Robotics Research, 5(4), 56-68 (1986).

[11] Zatsiorsky VM: Kinematics of human motion, Human Kinematics Publishers Inc. (1998). 